\title{
Cyber Bullying Scrutiny for Women Security in Social Media
}

\author{
Madhubala.D ${ }^{\mathrm{a}, 1}$, Rajendiran. $\mathrm{M}^{\mathrm{b}}$ and Elangovan. $\mathrm{D}^{\mathrm{b}}$, Priya. $\mathrm{R}^{\mathrm{c}}$ \\ ${ }^{\mathrm{a}, 1}$ PG Scholar, Dept of CSE, Panimalar Engineering College, Chennai \\ ${ }^{b}$ Professor, Dept of CSE, Panimalar Engineering College, Chennai \\ ${ }^{c}$ Assistant Professor, Dept of CSE, Panimalar Engineering College, Chennai
}

\begin{abstract}
In recent year, the number of users in social media has been increased multiple times when compared to the past. So most of them are aware about the current affairs in day today life. It is the medium for the user to express the opinion without facing any difficulty. At the same there will be a lot of bullying occurs in social media. The bullying like abuse word, aggressive text or posting some unwanted message. So the women feel unsecured in the society. Although a lot of techniques and methodology has been raised, but still the problem remain same. The major problem is the abuse word can be eliminated by the mean of report to the particular social media. In this methodology the unwanted message can be truncated in between the sender and receiver itself. So there other person cannot be affected in the cyberbullying. Also the unstructured data can be increased in the social media. So it leads to complex for analyzing the text. With the help of sentiment analysis, we can easily filter the unwanted text. Based on the criteria, the paper has been proposed with the problem statement as a main goal and developed a system in machine learning.
\end{abstract}

Keywords. Machine learning, Cyber bullying, socialmedia, Aggressiveword etc.

\section{Introduction}

Nowadays most of people use the social media in both positive way as well as negative ways .So most of them are aware about the current situation about the country, education or technology basis .The social media is an ideal platform for the youngster, because if there is any tweets about the organization, employment as well as the opportunity for exposing the ideas and also we can update the current technology and social activities. The users in social media can be increased by day -today life. However through the social media, user can freely express the view about the current situation. Similarly the most of the celebrity can actively interact the fans through the social media. In current trends most of them are actively participated in social media., Likewise the politician, business man, common people and other higher authority can also available in media. The social media platform consists of nearly millions of people .In the same manner, the negative ways are posting aggressive comment, offences in the form of abuse word. Based on threat, the women suffer a lot which

${ }^{1}$ D.Madhubala, PG Scholar, Dept of CSE, Panimalar Engineering College, Chennai - 600123;

E-mail: madhubaladakshina@gmail.com 
leads to psychology problems such as stress, mood swing and suicide can also occur. The main aim of this system is to provide the precautionary steps to prevent the women in social media platform. However, it also assists to analyses the threats against in social networking sites. The goal of this system is to filter the unwanted text which is against the women in the form of threats or violence's. With the help of the machine learning technique we can predict the text in social media.

\section{Related works}

Today social networking sites is the fast growing sites among the teens. So there is possibility for vulnerable to get exposed to bullying. In this paper they are discussed the supervised learning technique to detect cyberbullying. For feature detection ,they consider the two hypotheses to find the abuse comment or offensive comment .Based on the comment they faces the negative consequence. The initial experiment shows that the features using hypothesis .Furthermore with traditional extraction techniques like $\mathrm{N}$-gram,TF-IDF can be used for increasing the accuracy in the system.[1]

There is an increase in endangered text occurs among the young people in online [2]. The recent report demonstrate that cyberbullying is one of the major problem in social networking sites.In this paper, they focused the bullies, victims and bystanders in social media. In automatic cyberbullying, they performed the series of binary classification for better feasibility. Similarly they use the linear support vector machine for manipulating the feature set.

Vinita Nahar, Sanad Al-Maskari et al[3] has proposed that semi-supervised learning with fuzzy SVM algorithm. Since current approach is based detection can be static and also they find the difficulty to handle the imbalanced data efficiency and noisy. They consider the different evaluation scenarios to indicates the superiority of the proposed fuzzy SVM is better than all the existing method.The significant phenomenon on harassment by cyberbullying in social media. The existing system proposed that the three bottleneck approach [4]. The objective of the first approach is only on the social media platform. The second approach focus on address (like hashtag) just the topic based analysis can be done. And then the third approach is feature of the data which is already predefined. So the author proposed that the deep learning concept to overcome the bottle neck approach. . SemiuSalawu, Yulan He et al [5] suggested that the systematic review on cyber bullying detection on social networking site. Most of the research paper focused on the machine learning and natural language processing technique. In supervised learning mainly focus on the SVM and naive bayes classification and also in lexicon based uses the presences of word for categorize the bullying words. The challenging task is the combination of cross model with the various modalities and dependencies between the structural. So they proposed the XBully framework [6]. Similarly the algorithm are naive bayes, Support Vector Machine , JRIP is RIPPER Algorithm ,Projective Adaptive Resonance Theory ,KNearest Neighbor, Decision Tree, Logistic Regression, Artificial Neural Network for identification and detection for cyber bullying content. And also the accuracy can be increased by F-measure [7]. The social media users has been increasing day by day .So there is a harmful threat message has been received by user [8]. It mainly cause the negative impact on the person's life and also even society faces the teen suicides. However the author proposed the automatic detection of harmful message in social 
media. It is based on word embedding and also we can expand the list of predefined insulting word[16-30].

\section{Methodology}

The below Fig.1 depicts the detection of cyberbullying text. First we need to load the cyberbullying dataset. After that the dataset can be preprocessed .In preprocessing step, it involve the tokenization of the text, removal of special character and stemming. Then we need to extract the features from preprocessing. Finally ,it involve the machine learning algorithm and then we need to integrated with twitter API .At last it remove the harmful tweet against women.Generally social media allow the people to share the information and provide effective communication among the society. At the same time, it as the negative side for the women such as abuse words and also posting some bad comment against women. So they suffer a lot and also even lead to the negative impact on the personal life. Threats can also occur from normal women to the celebrity women. The abuse word and aggressive word is generally referred as a Cyberbullying .In digital communication, the message can be send by the individual or group of the people perform the cyberbullying activities against the victim.

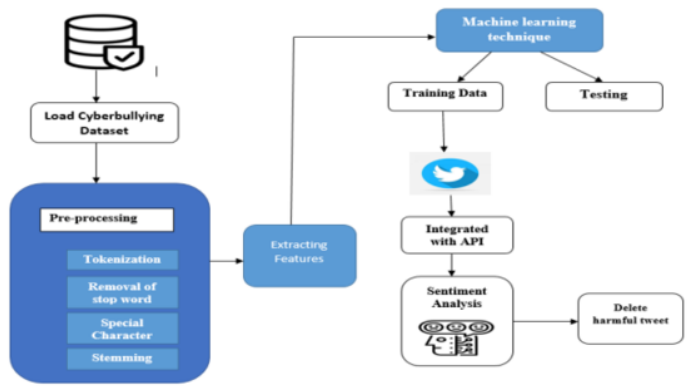

Figure 1. Architectural diagram

\section{Data Flow Diagram}

\subsection{Level 0}

This diagram represents that extracted text in twitter (input.txt) is given as an input to the Twitter classification where the system is already trained to detect cyberbullying dataset from the text and gives the result based on training given to the system.

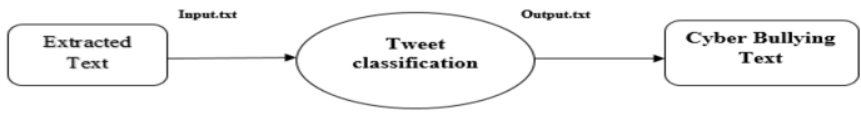

Figure 2. Level 0 DFD Diagram 


\subsection{Level 1}

The Cyber Bullying text is given as an input to the system and text is given into the pre-processing step .Then the text is tokenized and then integrated into the twitter API which can detect the threats or abuse words in social media.

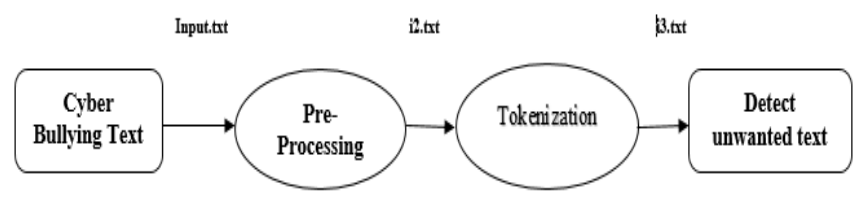

Figure 3. Level 1 DFD Diagram

\section{Conclusion and future work}

In this paper, we have collected several data set for detecting the cyberbullying text. This methodology of collecting opinions of people from social media enables a quantitative information across the globe including the rate of their sentimental feelings on harassment, providing a strong base for improving and empowering security. This way of analyzing sentimental feelings of public and providing guidelines in order to lessen insecure problems arising in the society would definitely help in reducing the risk of these nauseous activities acting upon society. This proposed system would recommend various guidelines for detecting aggressive word and precautionary efforts needs to be establish by the government and public awareness to save the woman from the various violence in the form of sexual harassments. This model also takes advantage to alert the woman from the difficult situations. Further, the system provide better efficiency when compared to other systems. And also it provide more safety to the women in an effective manner.

\section{References}

[1] Vikas S Chavan, Shylaja S S, Machine Learning Approach for Detection of Cyber-Aggressive Comments by Peers on Social Media Network, Proceeding of International Conference on Advances in Computing, Communications and Informatics (ICACCI),2015

[2] Cynthia Van Hee, Gilles Jacobs , Chris Emmery, Bart Desmet, ElsLefever, Ben Verhoeven, Guy De Pauw, Walter Daelemans and VéroniqueHoste, Automatic detection of cyberbullying in social media text ,Journal of PLOS one,2018.

[3] Vinita Nahar,Sanad Al-Maskari,Xue Li,Chaoyi Pang, Semi-supervised Learning for Cyberbullying Detection in Social Networks ,proceeding on $25^{\text {th }}$ Australasian Database Conference, 2014.

[4] Sweta Agrawal, Amit Awekar, Deep Learning for Detecting Cyberbullying across Multiple Social Media Platforms, Proceeding on European Conference on Information Retrieval, 2018.

[5] SemiuSalawu; Yulan He; Joanna Lumsden , Approaches to Automated Detection of Cyberbullying: A Survey ,Journal of IEEE Transaction on Affective Computing, Volume: 11,2020.

[6] Lu Cheng, Jundong Li, Yasin N. Silva, Deborah L. Hall,

[7] Akshi Kumar,Nitin Sachdeva, Cyberbullying Checker: Online Bully Content Detection Using Hybrid Supervised Learning, International Conference on Intelligent Computing and Smart Communication ,2019

[8] Rui Zhao, Anna Zhou, Kezhi Mao, Automatic detection of cyberbullying on social networks based on bullying features, Proceedings on 17th International Conference on Distributed Computing and Networking,2016. 
[9] Harsh Dan, Jundong Li .Huan Liu, Sentiment Informed Cyberbullying Detection in Social Media , Journal of Computer Science, 2017.

[10] Reynolds K, Kontostathis A, Edwards L, Using Machine Learning to Detect Cyberbullying, Machine Learning and Applications and Workshops (ICMLA),proceedings on 10th International Conference , vol.2, pp.241,244,2011.

[11] Nahar, V, Li, X, Pang, C, Zhang, Y, Cyberbullying detection based on text-stream classification , Proceedings of the Eleventh Australasian Data Mining Conference, 2013.

[12] Bretschneider U, Wöhner T and Peters R, Detecting online harassment in social networks, Proceeding on Thirty Fifth International Conference on Information Systems ,2014.

[13] Abeele MVand De Cock R, Cyberbullying by mobile phone among adolescents: The role of gender and peer group status, vol. 38, no. 1, pp. 107-118, 2013.

[14] Akshaya.B, M.Rajendiran, Securing Documents Using Digital Certificate-A Review. International Journal of Research in Advent Technology (IJRAT), 2018

[15] Lu Cheng, RuochengGuo, Yasin Silva, Deborah Hall and Huan Liu, Hierarchical Attention Networks for Cyberbullying Detection on the Instagram Social Network, Proceedings of SIAM International Conference on Data Mining,2019.

S.Hema Kumar, J.UdayKiran, V.D.AmbethKumar,G.Saranya, Ramalakshmi V, "Effective Online Medical Appointment System ”, International Journal of Scientific \& Technology Research, Volume 8, Issue 09 September 2019, Pages $803-805$.

[16] Ambeth Kumar.V.D, Dr.M.Ramakrishnan, V.D.Ashok Kumar and Dr.S.Malathi (2015) "Performance Improvement using an Automation System for Recognition of Multiple Parametric Features based on Human Footprint", kuwait journal of science, Vol 42, No 1 (2015), pp:109-132.

[17] Ambeth Kumar.V.D, V.D.Ashok Kumar, S.Malathi, K.Vengatesan, M.Ramakrishnan .Facial Recognition System For Suspect Identification Using A Surveillance Camera.Pattern Recognition and Image Analysis (Springer), Volume 28, Issue 3, pp 410-420, 2018. 10.1134/S1054661818030136)

[18] Ambeth Kumar.V.D and M.Ramakrishan .Employment Of Footprint Recognition System. in the month of December for Indian Journal of Computer Science and Engineering (IJCSE) Vol. 3 No.6 Dec 2013

[19] Ramya,T.,Dr.Malathi,S.,ratheeksha,G.R. and Dr.V.D.Ambeth Kumar (2014). Personalized authentication procedure for restricted web service access in mobile phones. Applications of Digital Information and Web Technologies (ICADIWT), 2014, Page(s):69 - 74, Bangalore, India (ISBN:978-14799-2258-1)

[20] V.D.Ambeth Kumar (2018) . Novel Wireless Sensing System For The Welfare Of Sewer Laborers. Healthcare Technology Letters (IET) Volume 5, Issue 4, p. $107-112$.

[21] V.D.Ambeth Kumar, G.Saranya , D.Elangovan, V.RahulChiranjeevi, V.D.Ashok Kumar, "IOT Based Smart Museum Using Wearable Device", Lecture Notes in Networks and Systems, Vol.55, pp: 33-42, 2018

[22] V.D.Ambeth Kumar and Dr.M.Ramakrishan .Footprint Based Recognition System.in the month of April for the International Journal Communication in Computer and Information System, Volume 147, Part 3, 358-367, DOI: 10.1007/978-3-642-20573-6_63, April 2011

[23] V.D.Ambeth Kumar (2017).Efficient Routing for Low Rate Wireless Network a Novel Approach. International Journal of Image Mining, Vol. 2, Nos. 3/4, 2017, 2017

[24] V.D.Ambeth Kumar, V.D.Ashok Kumar, Dr.S.Malathi and P.Jagaeedesh, (2014).Intruder Identification using Footprint Recognition with PCA and SVM Classifiers.for the International Journal of Advanced $\begin{array}{lllllll}\text { Materials Research } & \text { Vols.1345, PP } & \text { 984-985 } & \text { (2014) } & \text { pp }\end{array}$ [DOI:10.4028/www.scientific.net/AMR.984-985.1345]

[25] V.D.Ambeth Kumar, G.Gokul, S.Malathi, K.Vengatesan, D.Elangovan, B.Chitra .Implementation Of The Pulse Rhythemic Rate For The Efficient Diagonising Of The Heart Beat. Healthcare Technology Letters (IET) 2019 Apr 17;6(2):48-52.

[26] S.V. Ruphitha et.al . Management of Major Postpartum Haemorrhage by using Zigbee protocol - A Review .2021 6th International Conference on Inventive Computation Technologies (ICICT) (DOI: 10.1109/ICICT50816.2021.9358757)

[27] Ambeth Kumar.V.D, Dr.M.Ramakrishnan, V.D.Ashok Kumar and Dr.S.Malathi (2015).Performance Improvement using an Automation System for Recognition of Multiple Parametric Features based on Human Footprint. kuwait journal of science, Vol 42, No 1 (2015), pp:109-132.

[28] Rajamani.V, S Murugavalli, “ A High Speed Parrallel Fuzzy C-means algorithm for Tumor Segmentation", ICGST International Journal on BIME 6 (1), 2006

[29] Dhivya.M, K Valarmathi .IoT Based Smart Electric Meter .Lecture Notes on Data Engineering and Communication Technologies, volume 35, pp 1260-1269, 2020. 